\title{
A Simple Neuron Servo
}

\author{
Stephen P. DeWeerth, Student Member, IEEE, Lars Nielsen, Carver A. Mead, and Karl J. Åström, Fellow, IEEE
}

\begin{abstract}
This paper describes a simple servo controller build from components having neuronlike features. Experimental results illustrate the properties of the system, and a comparison is made with conventional controllers.
\end{abstract}

\section{INTRODUCTION}

B OLOGICAL systems are currently popular sources of inspiration in the engineering community. Biological motor systems can perform very gentle and precise motions, although the individual components such as neurons and muscles are imprecise. This is accomplished by using highly redundant systems that greatly improve accuracy and fault tolerance. Conventional servos, on the other hand, are typically built from high-precision components. In spite of this, they can only mimic the dexterity of a human hand in very restricted situations

One important aspect of biological systems is that information is transferred via nerve pulses. This representation has several useful features. Pulses are used when controlling muscles, which are complicated mechanical actuators with both damping and internal friction [1], [2]. The advantages of using pulses when driving friction-limited systems are well known and will be illustrated in Section III of this paper.

Current VLSI implementations of neural systems 13] offer practical solutions to engineering problems. This paper will demonstrate that these VLSI devices are well suited for the implementation of servo controllers. Since the controllers require only a small number of circuit elements, the systems require a silicon surface which is many orders of magnitude smaller than a conventional system with A/D, CPU, memory, and D/A units. The system can be made very reliable, for example, by duplicating the circuit elements, so that a component failure will only lead to a slight degradation in the system performance. Apart from these advantages related to cost and function, the systems are easy to integrate with sensors and actuators.

The circuits used are described in Section II and it is shown how they can be combined with a proportional-derivative (PD) controller. Section III gives experimental results, and the conclusions are given in Section IV.

\section{System Implementation}

The design methodology introduced by Mead [3] uses analog integrated circuits which operate primarily in the subthreshold region of the transistor characteristics. This approach has several advantages, namely small physical size, low power consumption, and large signal range. This means that vast computations can be performed on a small silicon area consuming

Manuscript received August 12, 1990; revised November 19, 1990

S. P. DeWeerth and C. A. Mead are with the Department of Computer Science, California Institute of Technology, Pasadena, CA 91125.

L. Nielsen and K. J. Åström are with the Department of Automatic Control, Lund Institute of Technology, Box 118, 22100 Lund, Sweden.

IEEE Log Number 9042080. only a few milliwatts. Another advantage is that both currents and voltages are used as signal types. The basic circuits are described in [3]. Some modifications of these have been carried out, and these are described in detail in [4]. Some additional circuit elements have also been implemented. For example, a circuit with the characteristic

$$
i=\sum_{n=1}^{N} i_{b_{n}} \tanh \left(\kappa \frac{V_{1_{\mathrm{n}}}-V_{2_{\mathrm{n}}}}{2 V_{t}}\right)
$$

has been implemented (see Fig. 1). In this equation, $V_{t}$ is the thermal voltage and $\kappa$ the gate voltage efficiency; $i_{b_{n}}$ denotes the the bias current and $\left(V_{1_{n}}-V_{2_{n}}\right)$ the differential input to the $n$th circuit element. The appearance to the tanh-function is due to the exponential transistor characteristics in the subthreshold region. Notice that for small voltage differences (1) approximates to

$$
i=\sum_{n=1}^{N} k_{n}\left(V_{1_{n}}-V_{2_{n}}\right)
$$

where $k_{n}=i_{b_{n}} \kappa /\left(2 V_{t}\right)$. The system of Fig. 1 is a useful component when implementing control systems since it is a key element both in simple controllers such as PID and in state space controllers [5]. The circuit is also useful for implementation of neural networks. It follows from (1) and (2) that the circuit acts like a summation unit with saturation arithmetic.

The circuit is implemented using an array of wide-range transconductance amplifiers similar to [3, fig. 5.11]; these are modified to be dual-output [4, fig. 2] so that each amplifier generates a pair of complimentary output currents that are summed onto two global wires. The input to each element of the array is encoded in the diffential voltage presented to the amplifier $\left(V_{1_{n}}-V_{2_{n}}\right)$. The input gain is determined by the amplifier bias voltage $V_{b_{n}}$, which sets the bias current $i_{b_{n}}$. The resulting aggregated currents are half-wave rectified and converted into pulses by a pair of neuron circuits [3, fig. 12.3]. The complete implementation is shown here in Fig. 1.

This circuitry mimics a naive neuron model. It converts an arbitrary number of weighted differential voltages into a pair of pulse trains. For small differential input voltages, the amplifiers are approximately linear but they saturate for large signals. The neuron circuits also produce a linear transformation from their dc input currents to the duty cycle of their output pulse trains. The dual-rail, complementary nature of the system guarantees nonoverlapping pulses. The sign of the aggregated currents determines which neuron circuit is firing. The pulse outputs from the chip can be used as inputs of the two terminals of a dc motor to bidirectionally control the motion of a mechanical system.

\section{The Proportional-Derivative Controller}

The structure in Fig. 1 is used to implement the proportionalderivative controller shown in Fig. 2. The proportional section is implemented by using a single dual-output transconductance 


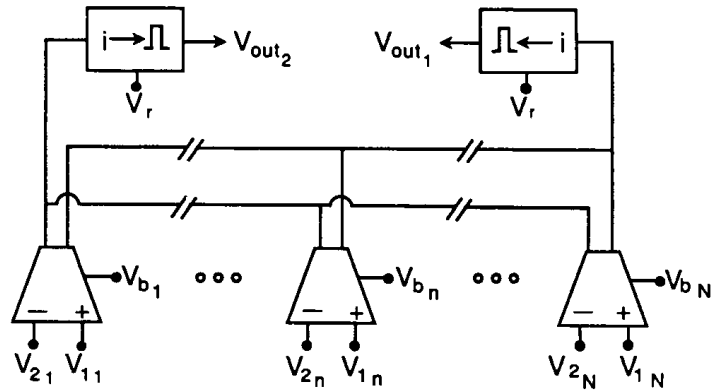

Fig. 1. VLSI circuit that mimics simple neuron behavior.

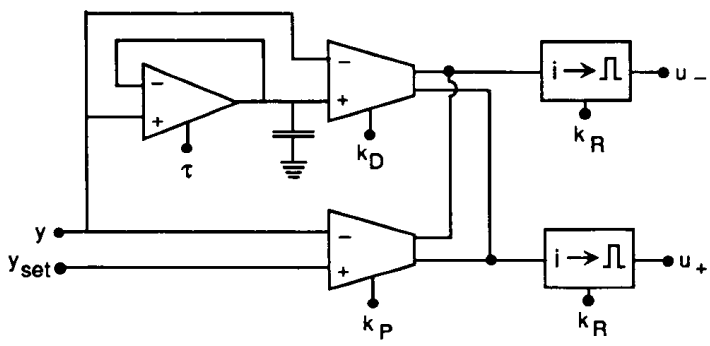

Fig. 2. P-D controller implemented using the circuit in Fig. 1 with a widerange dual-output differentiator as one of its two elements.

amplifier. The derivative section is implemented using a differentiator circuit [3, fig. 10.5] where the output amplifier is replaced by a dual-output amplifier. The inputs to the system are the operating point represented as a voltage $y$, and a set-point reference voltage $y_{\text {set. }}$. The constant $\tau$ sets the time constant of the differentiator. The constants $k_{P}, k_{D}$, and $k_{R}$ are used to set the gains for the proportional and derivative sections of the controller. The outputs of the controller are

$$
\begin{aligned}
& u_{+}=\frac{k_{P}}{k_{R}}\left(y_{r}-y\right)-\frac{k_{D} \tau}{k_{R}} \frac{d y}{d t} \\
& u_{-}=\frac{k_{P}}{k_{R}}\left(y-y_{r}\right)+\frac{k_{D} \tau}{k_{R}} \frac{d y}{d t} .
\end{aligned}
$$

In this system the parameters $k_{P} / k_{R}$ and $k_{D} \tau / k_{R}$ have to be set externally. A natural extension is to set these parameters adaptively [6].

\section{EXPERIMENTAL RESULTS}

A number of controller chips have been manufactured. They have been tested to control a motor drive system. In this way we have obtained a feel for the variability of the circuits and the performance of the controller. The particular system used in the experiments was an $18 \mathrm{~V}$ dc motor having a gear box with ratio $1: 8$, a coupling with some backlash, and an inertial load. The dc servo used has a velocity range of $0-100 \mathrm{rad} / \mathrm{s}$. More details can be found in [4].

The neuron servo was compared with a conventional analog controller. At medium and high speeds both controllers work well. However, at very slow speeds, the neuron controller continuees to drive the system while the conventional controller fails. The conventional controller needs a fairly high reference signal to cause the motor to break free of the static friction. When the reference velocity is decreased, the system has a ten-
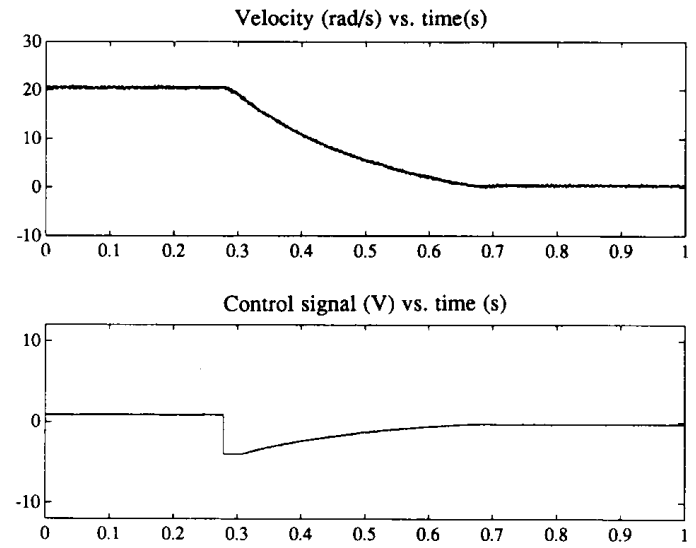

Fig. 3. Step response using the conventional servo. The desired velocity is changed from a medium high speed, $20 \mathrm{rad} / \mathrm{s}$, to $0.1 \mathrm{rad} / \mathrm{s}$. The steadystate control signal is not sufficient to create any motion.

dency to stick. Adding integral action gives rise to the wellknown stick-slip phenomenon. With the pulse servo, however, each pulse contains enough energy for the motor to overcome the static friction. Thus, instead of stopping when the reference velocity is small, the motor continues to move. At high speeds the time-averaged output of the pulse controller operates as the control signal, and the duty cycle controls the speed, as expected. At low velocities, however, each pulse generates a discrete movement of the motor, similar to the operation of a stepper motor. The conventional controller can hardly function at speeds below $1 \mathrm{rad} / \mathrm{s}$. At these speeds a small disturbance can stop the motion. The neuron servo, on the other hand, can work at speeds below $0.1 \mathrm{rad} / \mathrm{s}$.

Detailed experiments comparing the responses of the neuron servo with a conventional controller will now be presented. The controller gains were tuned so that the response times were approximately the same for the neuron controller and the conventional controller. The desired velocity was changed from a medium high speed, $20 \mathrm{rad} / \mathrm{s}$, to a desired velocity, $0.1 \mathrm{rad} / \mathrm{s}$, which is an order of magnitude below the sticktion limit.

Fig. 3 shows the response with the conventional controller. The system sticks when the velocity approaches $1 \mathrm{rad} / \mathrm{s}$. Fig. 4 shows the same experiment with the neuron controller, which controls the system well into the sticktion region. Fig. 5 shows an experiment with the neuron servo having the set point 0.1 $\mathrm{rad} / \mathrm{s}$. The figure shows that the servo works in this region, which is an order of magnitude below the sticktion limit. The conventional servo does not move at all in the corresponding situation.

\section{Conclusions}

This paper reports initial progress in a project whose goal is to use silicon neurons as components for implementing control systems. The basic circuit elements are described. A key element is a component with neuron like capability that takes voltages as inputs and generates a pulse train as the output. It is shown how the circuits are combined to a proportional and derivative controller. The advantages of using a pulsed output representation to improve slow-speed operation of a friction-limited system is demonstrated. The utility of exploiting parallelism, aggregation, and redundancy to improve system- 

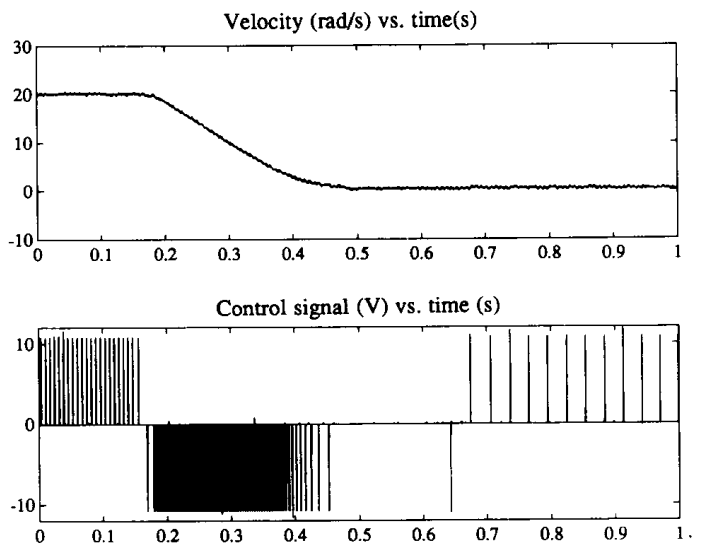

Fig. 4. Step response using neuron servo. The desired velocity is changed from a medium high speed, $20 \mathrm{rad} / \mathrm{s}$, to $0.1 \mathrm{rad} / \mathrm{s}$. The control is obtained by changing the pulse frequency, and the motion continues also at the low speed.
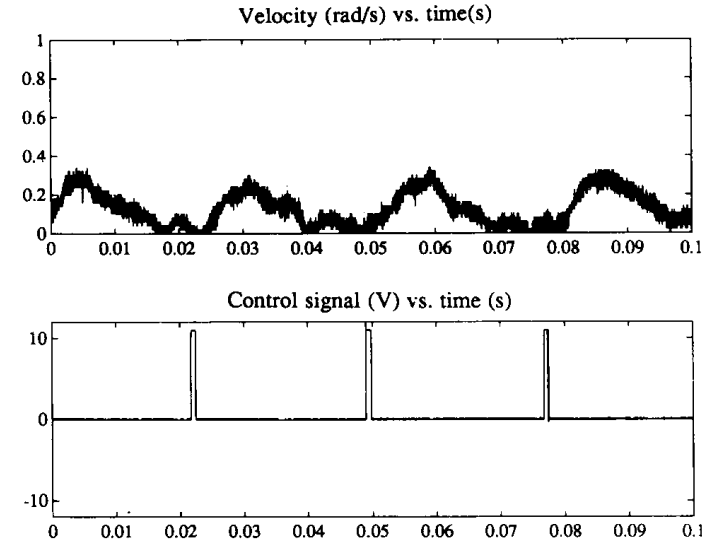

Fig. 5. Experiment with the neuron servo at a velocity $0.1 \mathrm{rad} / \mathrm{s}$, for the dc servo. (The conventional servo does not move in the corresponding situation.) Each pulse moves the servo a bit to create a time-averaged velocity of the desired value.

level performance given imprecise low-level components has also been discussed.

One advantage of the analog VLSI technology used in this implementation is the ease of combining sensing, intermediate processing, and control (both conventional and adaptive). Since the control circuitry is implemented using VLSI, it can easily be integrated on the same chip as these sensory systems to form integrated sensory systems. Such integrated sensorimotor systems have previously been presented [7], [8]. In the systems we have built so far the controller parameters have to be set externally. Adding adaptation [6] is a natural and challenging extension.

\section{REFERENCES}

[1] R. C. Woledge, N. A. Curtin, and E. Homser, Energetic Aspects of Muscle Contraction, Monographs of the Physiological Society, No. 41,1985 .

[2] K. A. P. Edman and F. Lou, "Changes in force and stiffness induced by fatigue and intracellular acidification in frog muscle fiber," J. Physiol., vol. 424, 133-149, 1990.

[3] C. A. Mead, Analog VLSI and Neural Systems. Reading, MA: Addison-Wesley, 1989.
[4] DeWeerth, S., L. Nielsen, C. Mead, and K. Åström, "A neuronbased pulse servo for motion control," in Proc. IEEE Int. Conf. Robotics and Automation, (Cincinnati, OH), 1990, pp. $1698-1703$.

[5] K. J. Àström and B. Wittenmark, Computer Controlled Systems. Englewood Cliffs, NJ: Prentice Hall, 1990.

[6] K. J. Åström and B. Wittenmark, Adaptive Control. Reading, . MA: Addison-Wesley, 1989.

[7] S. P. DeWeerth and C. A. Mead, "A two-dimensional visual tracking array," presented at Advanced Research in VLSI, Fifth MIT Conf., Mar. 1988.

[8] S. P. DeWeerth and C. A. Mead, "A VLSI model of adaptation in the vestibulo-ocular reflex," Advances in Neural Information Processing Systems, vol. 2, D. S. Touretzky, Ed. Los Altos, CA: Morgan Kaufmann, 1990.

Stephen P. DeWeerth ( $\mathbf{S}^{\prime} \mathbf{9 0}$ ) received the B.A. degree in mathematics and chemistry from Wartburg College, Waverly, IA, in 1985, and the M.S. degree in computer science from the California Institute of Technology, Pasadena, in 1987. He is currently a graduate student in computation and neural systems at the California Institute of Technology. His research interests include analog VLSI circuitry and electromechanical systems applied to robotics. 


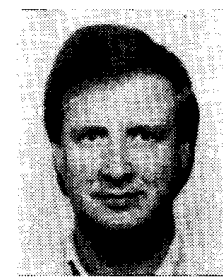

Lars Nielsen was born in Sweden in 1955. He received the $M$.Sc. degree in engineering physics in 1979 and the Ph.D. in automatic control in 1985, both from the Lund Institute of Technology.

$\mathrm{He}$ is currently Associate Professor in the Department of Automatic Control, Lund Institute of Technology, where he is responsible for a laboratory devoted to robotics and sensory control.

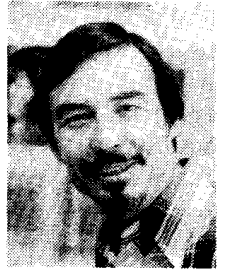

Carver A. Mead, Gordon and Betty Moore Professor of Computer Science, has taught at the California Institute of Technology for more than 30 years. He has contributed in the fields of solid-state electronics and the management of complexity in the design of very large scale integrated circuits, and has been active in the development of innovative design methodologies for VLSI. He has written, with Lynn Conway, the standard text for VLSI design, Introduction to VLSI Systems. His recent work is concerned with modeling neuronal structures, such as the retina and the cochlea, using analog VLSI systems. His new book on this topic, Analog VLSI and Neural Systems, has recently been published by Addison-Wesley.

Prof. Mead is a member of the National Academy of Sciences and the National Academy of Engineering, a foreign member of the Royal Swedish Academy of Engineering Sciences, a Fellow of the American
Physical Society, and Life Fellow of the Franklin Institute. He is also the recipient of a number of awards, including the centennial medal of the IEEE.

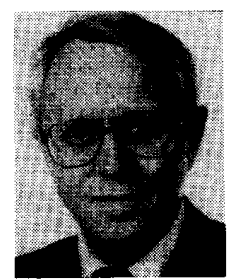

Karl J. Åström (M’71 -SM'77-F'79) was educated at the Royal Institute of Technology (KTH), Stockholm, where he has held various teaching appointments. He received Docteur Honoris Causa from l'Institut National Polytechnique de Grenoble in 1987.

He has been Professor of Automatic Control at Lund Institute of Technology since 1965. Before that he worked for IBM and the Research Institute of National Defense in Stockholm. His research interests cover broad aspects of automatic control, stochastic control, system identification, adaptive control, computer control, and computer-aided control engineering. He has published several books, among them Introductions to Stochastic Control Theory, Computer Controlled Systems-Theory and Design (coauthor, B. Wittenmark), Automatic Tuning of PID Controllers (coauthor, T. Hägglund), and Adaptive Control (coauthor, B. Wittenmark). He has contributed to several other books and has written many papers. He has supervised $28 \mathrm{Ph}$. D. students and numerous M.Sc. students. He holds three patents.

Dr. Asström is a member of the Royal Swedish Academy of Sciences, the Swedish Academy of Engineering Sciences (IVA), and the Royal Physiographical Society. He has received several awards, among them the Rufus Oldenburger Medal, the Quazza medal, and the IEEE Control Systems Science and Engineering Award. He is married to Bia Åström and they have two children, Karin and Kalle. His personal interests include sailing and skiing. 